\title{
Six Sigma application for quality improvement of the hot press in process
}

\author{
Xin $\mathrm{Li}^{*}$ \\ Institute of Management, Tianjin University of Technology, Tianjin, China
}

\begin{abstract}
Six Sigma is a systematic improvement method, a management approach designed to continuously improve corporate business processes and achieve customer requirement. This paper presents a case study conducted at a cell phone manufacturing company that intended to use the DMAIC method to carry out a structured approach to Six Sigma projects with fewer defective units generated by the cell phone hot press in process. In this process, Boss cracking and torque force failure led to high scrap costs and line downtime, which ultimately affected the supply of the assembly line. To this end, the Six Sigma approach was applied starting with the problem being defined, measured, and exhaustively analyzed to determine the root cause: poor injection molding structure, metal insert not perpendicular to the hole, improperly set molding machine parameters, and core/cavity dimension variation. A series of improvement measures addressing these factors increased the quality level of the process by improving the mold design to increase venting, optimizing the product structure to add the guide chamfer, and developing a standardized molding parameter setup procedure. Methods to maintain process control were also identified and implemented. The use of several quality tools and the use of Six Sigma methods also made the process more consistent.
\end{abstract}

\section{Introduction}

Due to constant changes in customer needs, new markets, innovations and other external factors, organizations are under constant pressure to improve the quality of their existing processes and to develop new processes to meet market trends. For this purpose, powerful methods of quality management, such as Six Sigma [1][2][3], can be used. According to Linderman et al [4], Six Sigma can be seen as a systematic, organized, and customer-oriented approach that aims to improve the performance and quality of processes, products, and services by using statistical techniques and scientific methods to analyze data and make decisions. The application of Six Sigma to repetitive processes has brought significant benefits to companies. This paper describes a project undertaken by a cell phone manufacturing company whose goal was to use Six Sigma methods to reduce the number of defective cells in a process that is a common part of cell phone manufacturing: the hot press in process. It has been found that in the hot press in process, if the process parameters are not set properly there are cosmetic defects such as failure of torque force or even cracking and higher scrap costs and reduced productivity, which affects the supply of the final assembly line. Through this project, we intend to reduce the cost of quality in this process and increase the number of products that are produced in the first time, thus not affecting the supply of the final assembly line. In this paper, we show the significant advantages obtained in the hot press in process by applying the DMAIC [5]approach and quality tools of the Six Sigma methodology. The paper is organized as follows. Section 2 describes the hot press in process. In section 3 , the project is reported according to the five steps of the DMAIC method. The final section presents the conclusions of the implementation of the methodology in the hot press in process.

\section{Hot Press in Process}

The cell phone manufacturing process is divided into ten stages according to the finished form of material processing. The first stage to fifth stage is mechanical parts manufacturing and simple assemblyng plastic parts; the sixth stage is the manufacturing of the motherboard and other small cards ; the tenth stage is the system integration and final test including installation of software packages, such as operating system software installation, operating system testing, and finally packaging and labeling. The hot press in process is in the first to fifth stage which is based on the high temperature heating state of the molded Boss feature for secondary heating, high temperature to change the physical properties of plastic, then use external force to press the brass insert with thread, natural cooling resin wrapped thread to form a stable structure. This series of processes is completed by a special hot machine. The Boss feature with press in brass insert is used for the assembly and fixing of cell phone body and cover, as the excellent physical properties of the brass inserts provide better mechanical strength and repeated use.

\footnotetext{
* Corresponding author: lynlee@stud.tjut.edu.cn
} 


\section{Six Sigma Project}

The Six Sigma project is presented next following the DMAIC steps.

\subsection{Define}

In the first phase, the project team was identified. The team consisted of a team leader, a black belt from the engineering department, process owner, process factory specialist, process maintenance specialist, two quality leaders and a sponsor. After the project team was selected, the following steps were performed.

Formalize the Six Sigma project by defining the name of the project, the time period of the data to be analyzed and the schedule of the work to be performed. The expectations of the project were exposed, especially in terms of the benefits to the company. A project charter containing this information is published.

Project Schedule Plan. The project schedule is particularly important in a quality improvement project. The ability to carry out the various elements of work effectively and to meet expectations is critical to the success of this project. In order to clearly understand the start and end time of each task and to better control and improve the project schedule, this paper uses Gantt charts[6] to plot the schedule control milestone nodes and the start and completion time of each phase of the task

Process characteristics. The SIPOC[7] diagram is used to identify the inputs that may affect the hot press in process and the outputs that need improvement. This tool allows focusing on the factors that lead to process changes.

Definition of project indicators. Defects were considered to be torque force that exceeded the upper and lower specification limits and poor appearance of cracked Boss columns. A number of other metrics were also used, such as the number of phone cases and the associated loss of scrap costs

Calculation of Sigma levels. The initial sigma level was calculated based on data from a 6-month time interval. The initial value was 2.53 . In order for the project to be considered successful, the goal to be achieved was to reduce the DPMO (defects per million opportunities) by at least $80 \%$, which corresponds to a sigma level of 3.38 .

\subsection{Measurement}

Correct data is the result to be obtained from the measurement phase of the work process. The correctness of the data depends on the reliability of the quality of the measurement process, i.e. the quality of the measurement system. Therefore, before analyzing the data, the reliability of the data source should be ensured, i.e., the reliability of the current exterior inspectors' judgment of the Boss feature cracking problem and the accuracy of the measurement of the torque force by the measuring personnel should be assessed. Because the data for these two indicators are attribute data and variable data, we used Assessment Agreement and Gage R\&R[8] analysis for these two parts of the measurement system, respectively. For the Boss feature cracking problem, we mixed the cracked phone top cover and the intact phone top cover with a good number and did visual identification by three different final inspectors, who were screened and specially trained. Each of them did 2 tests in order to verify the repeatability and reproducibility of the test. Samples and data were also collected for the torque force. Then, we did Assessment Agreement (Figure 1). and Gage R\&R analysis (Figure 2). on the collected attribute data and variable data, respectively, with the help of Minitab software. The experimental results prove that the current measurement system is acceptable.

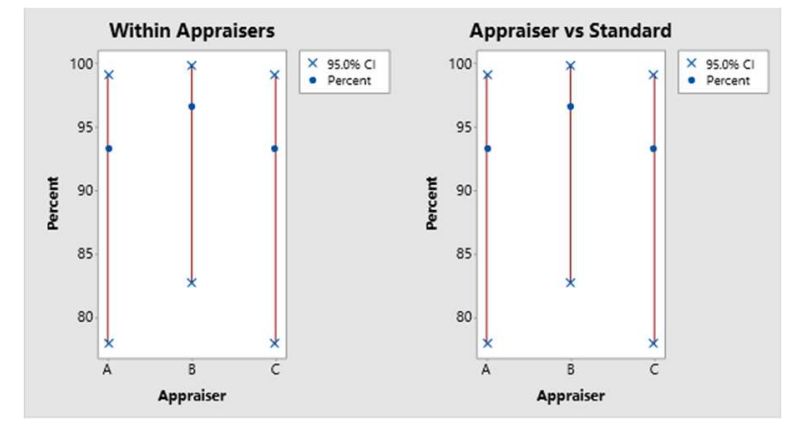

Figure 1. Schematic diagram of Assessment Agreement

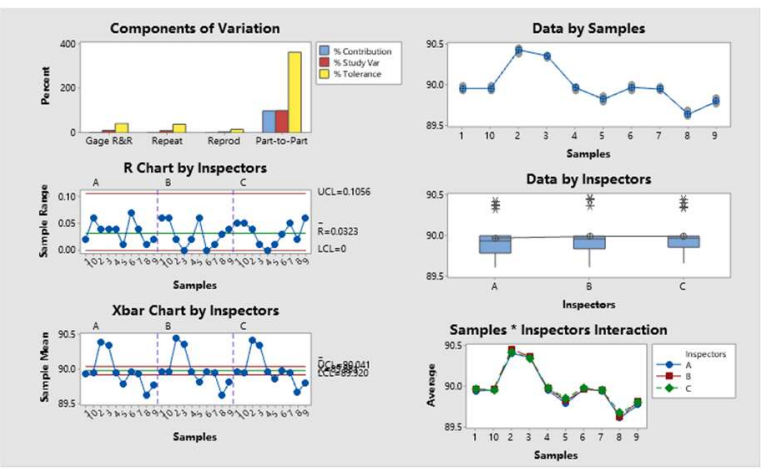

Figure 2. Schematic diagram of Gage R\&R analysis

\subsection{Analysis}

The analysis phase is mainly to determine the key factors affecting the process output through data analysis, i.e., to identify the key influencing factors of the process. In this paper, we mainly use cause-effect matrix diagram, failure mode analysis[9] and double ratio hypothesis testing. In order to understand the real causes of cracking and torque force failure in the process of hot press in of cell phones, team members used brainstorming method to start group discussion based on the process flow chart, and summarized the possible influencing factors from people, machine, material, method, environment and other impossible aspects after analysis, which appeared in the form of cause-effect matrix diagram. The input variables that may affect insert installation and hot press in were classified from the cause-effect matrix, and in order to further find the key factors caused by the defect, the input variables with high scores in the process of hot press in: temperature of the hot press in machine, material size, location of the hot press in fixture, strength of the material knit line and the method of installing the brass insert were 
analyzed with the FMEA to arrive at the RPN greater than 100 in Table 1. four critical factors. The team further confirmed that the four critical factors had an effect on Boss feature cracking and torque force failure by collecting data in small batches using a double ratio hypothesis test.

Table 1. Critical fators

\begin{tabular}{cc}
\hline Numbers & Factors description \\
\hline X1 & Poor injection molding structure \\
X2 & Metal insert not perpendicular to the hole \\
X3 & Improperly set molding machine \\
X4 & parameters \\
\hline
\end{tabular}

\subsection{Improvement}

Poor injection molding structure

Combined with the actual defective product occurrence status, the defective location of the model check, by comparing the cracking defective products within a week analysis found that the insert cracking mostly concentrated in one area. From the observation of the occurrence of the form, it is suspected that the location of the knit line, the need for mold flow analysis and short shot test mold analysis. Through the observation after mold flow analysis, the cracked area coincides with the location of the bond line we suspect, which will largely affect the strength and appearance of the product. For the weak structural strength at the bond line, we use cut through into the sub to improve the strength of the insert hole.

Metal insert not perpendicular to the hole

Before the improvement, the installation of copper nails only required to put the insert into the screw hole, after the improvement, we required to put the insert into the screw hole and press the insert downward to confirm the insert into place, and then carry out the hot press in operation at the next work station. And set the insert installation station as a key station, fixed staffing, by skilled operators to carry out special training and qualified to work. In addition, the manufacturing engineer will take the lead in designing and developing a positioning fixture with guiding function, so that the insert can be installed vertically even if the operator does not operate properly occasionally. Finally, from the product design point of view, we hope to add a chamfer with guiding function to the screw hole, and this plan needs to be sent back to the customer for confirmation before implementation.

Improperly set molding machine parameters

Through the analysis of the product of torque failure, as well as observation of the cracking of the cell phone that occurred many times during the assembly production process, we found that the main reason was the high temperature of the hot runner during the molding production and the non-standard operation during the production. After review the datasheet from raw material supplier, the manufacturing engineers set reasonable molding conditions: the molding machine material temperature should not be higher than 275 degrees Celsius. If you need to adjust the material temperature (more than
275 degrees Celsius), you must obtain the consent of the quality control worker before adjustment. When the machine is stopped for more than 3 minutes, the material must be cleared and the residual material in the hot runner must be eliminated.

Core/cavity dimension variation

In the process of mass production, the mold is used for a long time, the internal structure and parts wear, the size of the product in the corresponding position after the use of mold molding also has a corresponding variation. Reflected in this project is the variation of the size of the hole at the location of the hot insert. According to past experience, the dimensions guaranteed by the mold are often not included in the SIP process inspection specification, which eventually led to the problematic products output to the assembly line, resulting in torque force cracking after the insert was hot press in. The quality engineer designed and made the Go no Go inspection jig according to the customer specifications.

\subsection{Control}

Standardize the improved process settings

Develop standardized inspection specifications, add them to SIP for standardized control according to the improvement content set in the improvement phase, and issue the revised standard process inspection specifications to each inspector.

Use the statistical process control to monitor the factor level settings or the indicators of project

Process has been in a state of fluctuation, collect data from one month after implemented the improvement actions and draw the Figure 3 which is a P chart from SPC tools, all data are fluctuate in the mean line. The defects rate reduced to $97 \%$ and sigma level successful promote to 3.38 .

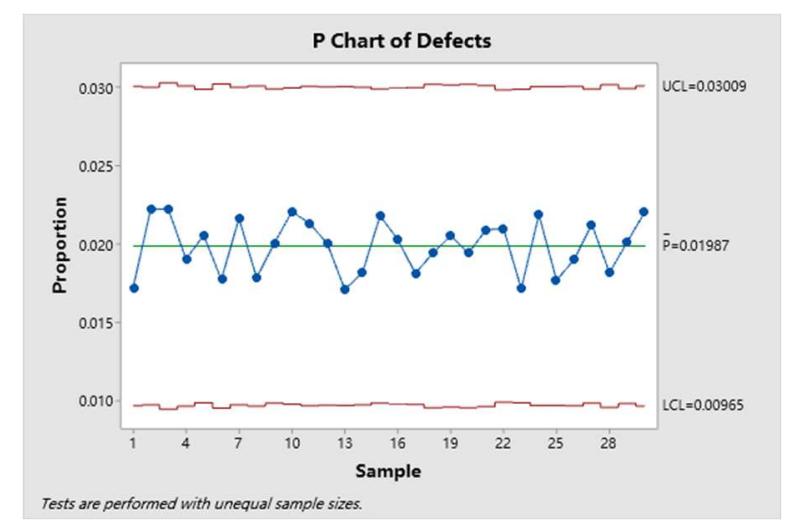

Figure 3. Schematic diagram of P Control Chart

\section{Conclusion}

This paper describes the completion of a Six Sigma project that consisted of reducing the number of defective units generated during the hot press in insert process for cell phones by applying Six Sigma methods to reduce the inherent cost of quality without affecting the supply of the final assembly line. 


\section{References}

1. J. Antony R. Banuelas. Design for Six Sigma: A Breadthrough Improvement Business Strategy for Achieving Competitive Advantage[J]. Manufacturing Engineering.2002, 81(1):24-26.

2. F. Breyfogle, Implementing six sigma: smarter solutions using statistical methods. John Wiley \& Sons, 2003.

3. M. Kumar, J. Antony, B. Cho, Project selection and its impact on the successful deployment of Six Sigma, Business Process Management Journal, 15(2009) 669-686.

4. Linderman K, Schroeder R G, Zaheer S, et al. Six Sigma: a goal-theoretic perspective[J]. Quality Control \& Applied Statistics, 2003, 21(2):193-203

5. Lenka Girmanová et al. Application of Six Sigma Using DMAIC Methodology in the Process of Product Quality Control in Metallurgical Operation[J]. Acta Technologica Agriculturae, 2017, 20(4) : 104-109.

6. Masahiro Shibuya. Production Planning and Management Using Gantt Charts[J]. Journal of Mechanics Engineering and Automation,2021,11(3):

7. Brown Charlie. Why and how to employ the SIPOC model.[J]. Journal of business continuity \& emergency planning, 2019, 12(3) : 198-210.

8. Jay Arthur. Gage R\&R[J]. Quality, 2017, 56(13) : 2021.

9. Lorenzi Clarice Inês and Ferreira Joao Carlos Espindola. Failure mapping using FMEA and A3 in engineering to order product development[J]. The International Journal of Quality \& Reliability Management, 2018, 35(7) : 1399-1422. 